\title{
Caracterización morfológica de tres procedencias de boldo (Peumus boldus) en una plantación joven de 6 años
}

\author{
Morphological characterization of three boldo (Peumus boldus) provenances, \\ in a 6 years old plantation
}

\author{
URSULA DOLL ${ }^{1}$, DARIO AEDO ORTIZ², PATRICIO LOPEZ CARRERA \\ ${ }^{1}$ Departamento de Gestión Forestal Ambiental, Universidad de Talca, Casilla 721-747, Talca, Chile. \\ E-mail: udoll@utalca.cl \\ ${ }^{2}$ Departamento de Producción Forestal, Universidad de Talca, Casilla 721-747, Talca, Chile. \\ E-mail: daedo@utalca.cl
}

\begin{abstract}
SUMMARY
Peumus boldus Mol., which belongs to the Monimiaceae family, is an evergreen endemic tree characteristic of the sclerophytic forest of the Chilean mediterranean region. Traditionally used as firewood and charcoal, this species usually simulates shrub characteristics, because of its ability to sprout from the trunk. The principal interest lays in the active compounds of a wide therapeutic use synthesized by its leaves. Boldo is wild harvested, which induced its domestication to reduce the disruption of natural ecosystems and improve the quality of the harvested products. The study objective was to morphologically characterize three boldo provenances, to determinate parameters which could be considered on future genetic selection. A series of morphological parameters were measured for individuals of the three origins cultivated at the Universidad de Talca. The morphological characteristics associated with plant architecture were principally influenced by the environment, while the characteristics associated with leaf morphology respond to genetic information modulated differentially by the environment.
\end{abstract}

Key words: Peumus boldus Mol., plant architecture, leaf morphology.

\section{RESUMEN}

Peumus boldus Mol., de la familia de las Monimiaceae, es un árbol siempreverde endémico, característico del bosque esclerófilo de la zona mediterránea de Chile. Usado tradicionalmente para leña y carbón, aparenta frecuentemente un hábito arbustivo, debido a su gran capacidad de rebrote. Su principal interés radica en los principios activos sintetizados en sus hojas, de amplio uso terapéutico. Actualmente la explotación del boldo se basa en la recolección silvestre, por lo que se inició su domesticación, con la finalidad de aminorar la presión sobre los ecosistemas naturales y mejorar la calidad de los productos cosechados. El objetivo del estudio se centró en caracterizar morfológicamente tres procedencias de boldo para determinar parámetros que podrían ser considerados en una posterior selección genética. A tal fin se cuantificó una serie de parámetros morfológicos sobre individuos de las tres procedencias cultivadas en el predio de la Universidad de Talca. Las características morfológicas asociadas a la arquitectura de la planta resultaron principalmente influenciadas por el medio ambiente, mientras que las características morfológicas de las hojas responden a información genética modulada diferencialmente por el ambiente.

Palabras clave: Peumus boldus Mol., arquitectura vegetal, morfología foliar. 


\section{INTRODUCCION}

El boldo (Peumus boldus Mol.) es un árbol siempreverde endémico de Chile, perteneciente a la familia de las Monimiaceae. Se distribuye entre las IV y X Regiones ( $30^{\circ}$ a $40^{\circ}$ latitud Sur) (1, $2)$, presentando una mayor abundancia en la zona mediterránea. Allí se mezcla con otros exponentes del bosque esclerófilo (Lithrea caustica, Quillaja saponaria y Cryptocarya alba), aunque tiende a formar bosques puros $(3,4)$.

Es una especie dioica que se caracteriza por su copa globosa, densamente ramificada, de color verde oscuro. Las hojas simples, opuestas, son de consistencia coriácea y muy aromáticas, como consecuencia de los aceites esenciales sintetizados por esta especie (5). La lámina es aovada a elíptica, con ápice obtuso y base redondeada (1). $\mathrm{Su}$ cara superior es de color verde oscuro y áspera al tacto, mientras que la cara inferior es verde amarillenta con nervaduras marcadas. El margen de las hojas es entero y revoluto cuando las mismas están expuestas al sol (5).

El boldo, que es usado tradicionalmente para leña y carbón, aparenta frecuentemente un hábito arbustivo, debido a su capacidad de rebrotar frente a la corta e incendios (3). El principal interés radica en sus hojas, que son cosechadas en verano para su comercialización y exportación $(6,7)$. Las hojas deshidratadas son utilizadas en la preparación de infusiones, a las que se les atribuyen propiedades de estimulantes de la digestión, colagogas, coleréticas y para afecciones hepáticas $(5,8)$. Los principios activos extraídos de sus hojas (aceites esenciales, alcaloides, flavonoides) han sido objeto de numerosas investigaciones que avalan su actividad biológica $(9,10,11,12,13)$.

Actualmente la explotación del boldo se basa en la recolección silvestre, por lo que se han iniciado estudios de domesticación y cultivo de esta especie con la finalidad de aminorar la presión sobre los ecosistemas naturales y lograr un mejoramiento de la calidad del producto cosechado $(14,15,16,17,18)$. Dentro de este contexto se estableció una plantación de boldos de tres procedencias, que ha sido evaluada durante varios años $(19,20,21)$. De estos estudios se concluye que las concentraciones de principios activos en hoja se comportan como caracteres cuantitativos, asociados a varios genes y cuya expresión es modificada por las condiciones ambientales (22), desta- cándose especialmente la concentración de aceites esenciales que arrojó una marcada diferencia entre procedencias. También se constataron diferencias significativas en la producción de principios activos entre las familias de un mismo origen (22), lo que permitiría una selección a favor de estos caracteres en futuros planes de mejoramiento. Los individuos de las diferentes procedencias de la plantación presentan además una coloración, tamaño de hoja y hábitos de crecimiento diferenciales, por lo que se planteó el siguiente estudio, con la finalidad de caracterizar morfológicamente las tres procedencias y determinar la sujeción de estos parámetros a un control genético o ambiental, para su posterior inclusión en planes de mejoramiento a favor de un aumento de la calidad del producto a cosechar.

\section{MATERIAL Y METODOS}

En una plantación de boldos descendientes de tres procedencias: Norte $\left(33^{\circ} \mathrm{S}\right.$, Cuesta La Dormida, Olmué), Centro $\left(35^{\circ} \mathrm{S}\right.$, Pencahue) y Sur $\left(38^{\circ} \mathrm{S}\right.$, Galvarino) (5), se seleccionaron 11 individuos por procedencia, siete de los cuales nunca fueron intervenidos, mientras que cuatro fueron podados en dos ocasiones (enero 2001 y agosto 2002), cortándose todos los ejes de la planta a una altura de $20 \mathrm{~cm}$ sobre el suelo.

La plantación fue establecida en junio 1998 sobre un suelo franco arcilloso en el Campus Lircay de la Universidad de Talca, con un diseño completamente al azar y a una densidad de 14.000 plantas por hectárea. El clima es mediterráneo templado, con una temperatura promedio de $14^{\circ} \mathrm{C}$ y $720 \mathrm{~mm}$ de precipitaciones, distribuidas en la época invernal (23). La plantación es suplementada con riego durante la época estival. Para su establecimiento se utilizaron plántulas provenientes de semillas cosechadas durante enero 1997 en su origen y sembradas en febrero del mismo año en contenedores de 0,4 litros, previo remojo por 48 horas en una solución de ácido giberélico (10 g/l). Desde su emergencia (mayo 1997) hasta su plantación (junio 1998) las plántulas fueron mantenidas en condiciones de invernadero, con riego frecuente y aplicaciones periódicas de fungicida (captan $1 \mathrm{~g} / \mathrm{l}$ ).

Desde enero hasta marzo de 2004, se efectuaron mediciones de características morfológicas sobre cada individuo seleccionado. 
Variables medidas. En los tres tallos de mayor longitud, individualizados como $1^{\text {er }}$ tallo dominante, $2^{\circ}$ tallo dominante y $3^{\text {er }}$ tallo dominante en orden decreciente, se midió:

- Largo total de tallo [cm].

En los $50 \mathrm{~cm}$ superiores de los tres tallos dominantes se cuantificó:

- Número de ramificaciones secundarias,

- Longitud de rama secundaria [cm] más larga,

- Número de nudos en tallo,

- Largo de internudos en tallo [cm], resultante del cuociente entre $50 \mathrm{~cm}$ y el Número de nudos en tallo,

- Número de nudos en la rama secundaria de mayor longitud,

- Largo de internudos en rama secundaria [cm], resultante del cuociente entre Longitud de rama secundaria y Número de nudos en rama secundaria,

- Número de hojas en la rama secundaria (incluye las hojas ubicadas sobre las ramificaciones de tercer grado).

En los tres tallos dominantes también se midió:

- Diámetro superior del tallo $[\mathrm{cm}]$ medido a 25 $\mathrm{cm}$ desde el ápice,

- Diámetro intermedio del tallo $[\mathrm{cm}]$ medido a la mitad del Largo total de tallo,

- Diámetro inferior del tallo $[\mathrm{cm}]$ medido a los $30 \mathrm{~cm}$ sobre el suelo.

Para cada individuo elegido se contabilizó:

- Número de rebrotes de tocón mayores a 0,3 $\mathrm{cm}$.

Para cuantificar las siguientes cuatro variables se cosecharon 12 hojas desarrolladas por individuo y se midió su largo $(L n)$ y ancho $(A n)[\mathrm{cm}]$ (este último a la mitad del largo respectivo), sin extender la hoja. Posteriormente las hojas fueron refrigeradas a $2^{\circ} \mathrm{C}$ por 20 días dentro de envases plásticos. Este tratamiento permitió extender las hojas evitando su quebradura.

- Ancho de hoja extendida (Ae) [cm],

- Largo de hoja extendida (Le) [cm],

- Enrollamiento en ancho de hoja [\%], calculado mediante la siguiente fórmula:

$$
\operatorname{Ancho}(\%)=\left[\frac{[A e-A n]}{A e}\right] * 100
$$

- Curvatura en largo de hoja [\%], calculado mediante la siguiente fórmula:

$$
\operatorname{Largo}(\%)=\left[\frac{[L e-L n]}{L e}\right] * 100
$$

Las siguientes cuatro variables fueron computadas en tres hojas desarrolladas de la exposición norte de cada individuo. En el laboratorio y con ayuda del FAN 3 de la tabla de colores publicada por la Royal Horticultural Society of London ${ }^{1}$, se determinó:

- Color del haz de las hojas,

- Color del envés de las hojas.

También en laboratorio y con la ayuda de una lupa binocular marca Meiji Tecno, modelo SKT 35613 (aumentos de 40X y 20X) se contabilizó en un área circular de $\pi \mathrm{mm}^{2}$ :

- Densidad de tricomas en el haz de las hojas $\left[\mathrm{n} \% \mathrm{~mm}^{2}\right]$,

- Densidad de tricomas en el envés de las hojas $\left[\mathrm{n} \% \mathrm{~mm}^{2}\right]$.

Diseño experimental y análisis de los datos. Se utilizó un modelo factorial de dos factores fijos, con datos no balanceados y proporcionales. El factor tratamiento consideró dos niveles: sin poda (SP) y con poda (CP); mientras que el factor procedencia consideró tres niveles: Norte $(\mathrm{N})$, Centro $(\mathrm{C})$ y Sur $(\mathrm{S})$.

Los resultados de cada variable se sometieron a un análisis de varianza bifactorial, para detectar la influencia de los factores tratamiento, procedencia y su interacción, sobre la variación total de los datos. Para interpretar los valores de $p$ arrojados por el análisis de varianza, se utilizaron los criterios sugeridos por Ramsey y Schafer (24), aceptándose como evidencia convincente $p \leq 0,01$ y evidencia moderada $0,01<p \leq 0,05$.

El contraste de medias se realizó mediante la prueba de Intervalos Múltiples de Duncan (25). En los casos en que el análisis de varianza mostró una interacción significativa $(p \leq 0,05)$ entre ambos factores, se realizó el contraste de medias, fijando el factor procedencia o tratamiento en cada

\footnotetext{
1 RHS Color Chart. The Royal Horticultural Society. London. 1995.
} 
uno de sus niveles. Para su evaluación, los datos expresados en porcentaje fueron modificados mediante la transformación angular o raíz cuadrada del arcoseno del valor (26).

Mediante el estadístico $X^{2}$ se verificó la existencia de asociación entre procedencias y las variables cualitativas Color del haz de las hojas y Color del envés de las hojas (27).

\section{RESULTADOS Y DISCUSION}

Las plantas estudiadas presentaban a simple vista un aspecto muy disímil, observándose una tendencia a presentar una coloración más oscura, un menor tamaño de hoja y una estructura más compacta en las procedencias Norte y Centro respecto de la procedencia Sur. Sin embargo las variables asociadas a la arquitectura de la planta, indicadas en el cuadro 1, no evidenciaron diferencias debido a la procedencia del material vegetal, en cambio sí mostraron influencia significativa del tratamiento de poda, en al menos un tallo dominante (cuadro 2). Esta misma tendencia se observó en la variable Número de rebrotes de tocón por individuo (cuadro 3), que sólo fue influenciada significativamente por el tratamiento de poda (cuadro 4). En general, los valores de las variables medidas en los individuos no podados superaron los valores obtenidos en los individuos podados. Sólo en el caso las variables Largo de internudo en tallo y Largo de internudo en rama secundaria (cuadro 1) se invierte esta relación, es decir, las plantas podadas superaron las no podadas, indicando un crecimiento en largo de los ejes caulinares en respuesta al tratamiento de poda.

La dinámica de crecimiento que adopta un árbol es expresión de su información genética interactuando con el medio ambiente, dando por resultado un determinado modelo de arquitectura (28). En este estudio las variables arquitectónicas consideradas resultaron ser influenciadas principalmente por el medio ambiente, ya que no se evidenciaron diferencias entre las procedencias. Sin embargo, el tratamiento de poda sí afectó la respuesta, pudiéndoselo interpretar como un tratamiento representativo del efecto ambiental. Sólo la variable Largo de internudos en rama secundaria resultó influenciada por la procedencia, o sea potencialmente asociada al genotipo de los individuos estudiados, con una evidencia moderada para los $1^{\text {os }}$ tallos dominantes y convincente para los $2^{\text {os }}$ tallos dominantes (cuadro 2). En el caso del $1^{\text {er }}$ tallo dominante, los Largos de internudos en rama secundaria de la procedencia Sur $(2,3$ y $2,6 \mathrm{~cm}$ para plantas sin poda y con poda respectivamente) superaron significativamente a los de las procedencias Norte y Centro $(1,6 ; 1,9$ y 2,3; $2,1 \mathrm{~cm}$ para plantas no podadas y podadas respectivamente), mientras que para el caso del $2^{\circ}$ tallo dominante la procedencia Sur con 2,3 y 2,6 $\mathrm{cm}$ superó significativamente a la procedencia Norte con 1,7 y $2,2 \mathrm{~cm}$ (cuadro 1 ). De esta manera esta variable da cuenta del aspecto más compacto que presentan en general las plantas de la procedencia Norte y Centro en la plantación.

El tratamiento de poda afectó el largo total de los ejes caulinares (Largo total de tallo y Longitud de rama secundaria, cuadro 1) y su diámetro (Diámetros superior, intermedio e inferior del tallo, cuadro 1), lo que podría traducirse en una menor incidencia de los tallos en el total de la biomasa producida por las plantas podadas (22), a pesar de que disminuye el número de hojas formadas.

El escenario cambia totalmente al considerar las variables cuantificadas en hoja (cuadro 3). En este caso en la mayor parte de los caracteres existe una interacción significativa entre la procedencia y el tratamiento o existe una influencia significativa del factor procedencia (cuadro 4). Sólo la variable Enrollamiento en ancho de la hoja es influenciada con evidencia convincente por el tratamiento de poda.

Generalmente la influencia de la procedencia se evidenció mediante una diferencia significativa entre la procedencia Norte y la procedencia Sur. El Ancho de la hoja extendida de las plantas no podadas de las procedencias Centro y Sur superó significativamente al de la procedencia Norte $(2,3$ y 2,2 contra $1,8 \mathrm{~cm}$ respectivamente), mientras que no hubo diferencias entre las plantas podadas (cuadro 3). El Largo de la hoja extendida fue significativamente diferente entre las tres procedencias en las plantas no podadas $(3,6,3,8$ y 4,1 $\mathrm{cm}$ en procedencias Centro, Norte y Sur respectivamente) y en las plantas podadas sólo difirió significativamente la procedencia Norte $(4,0 \mathrm{~cm})$ de la procedencia Sur (3,7 cm; cuadro 3). Analizando ambos caracteres conjuntamente, puede observarse que las dimensiones de hoja medidas dan cuenta del menor tamaño de hojas observado 


\section{CUADRO 1}

Respuestas medias de las variables medidas sobre los tres tallos dominantes de los individuos de cada procedencia $^{1}$.

Mean variable responses for the three dominant shoots from the individuals of each provenance ${ }^{2}$.

\begin{tabular}{|c|c|c|c|c|c|c|c|c|c|c|}
\hline \multirow{2}{*}{ Variable } & & \multicolumn{3}{|c|}{$1^{\mathrm{er}}$ tallo dominante } & \multicolumn{3}{|c|}{$2^{\circ}$ tallo dominante } & \multicolumn{3}{|c|}{$3^{\text {er }}$ tallo dominante } \\
\hline & & $\mathrm{N}^{3}$ & $\mathrm{C}$ & $\mathrm{S}$ & $\mathrm{N}$ & $\mathrm{C}$ & $\mathrm{S}$ & $\mathrm{N}$ & $\mathrm{C}$ & $\mathrm{S}$ \\
\hline \multirow{2}{*}{$\begin{array}{l}\text { Largo total de } \\
\text { tallo }(\mathrm{cm})\end{array}$} & $\mathrm{SP}^{4}$ & 129,3 & 146,0 & 104,7 & 117,3 & 131,4 & 98,6 & 111,1 & 120,9 & 80,7 \\
\hline & $\mathrm{CP}$ & 84,3 & 92,5 & 102,8 & 74,8 & 86,5 & 91,5 & 70,8 & 77,5 & 86,5 \\
\hline \multirow{2}{*}{$\begin{array}{l}\text { Número de } \\
\text { ramificaciones } \\
\text { secundarias }\end{array}$} & SP & 19,3 & 20,0 & 18,3 & 18,1 & 18,1 & 18,1 & 20,0 & 20,9 & 17,9 \\
\hline & $\mathrm{CP}$ & 16,3 & 17,0 & 21,5 & 13,0 & 16,8 & 19,0 & 13,5 & 12,5 & 19,3 \\
\hline \multirow{2}{*}{$\begin{array}{l}\text { Longitud de rama } \\
\text { secundaria }(\mathrm{cm})\end{array}$} & SP & 25,6 & 31,4 & 30,9 & 21,4 & 24,6 & 28,7 & 23,7 & 28,1 & 28,1 \\
\hline & $\mathrm{CP}$ & 22,3 & 19,5 & 23,0 & 20,8 & 26,0 & 20,5 & 16,3 & 16,5 & 17,0 \\
\hline \multirow{2}{*}{$\begin{array}{l}\text { Número de nudos } \\
\text { en tallo }\end{array}$} & SP & 19,7 & 18,6 & 20,6 & 18,6 & 18,6 & 19,3 & 20,4 & 17,7 & 18,4 \\
\hline & $\mathrm{CP}$ & 16,3 & 15,0 & 13,8 & 15,5 & 14,8 & 13,5 & 16,5 & 13,0 & 13,8 \\
\hline \multicolumn{2}{|c|}{ Largo de internudos SP } & 2,6 & 2,8 & 2,6 & 2,8 & 2,9 & 2,9 & 2,4 & 2,9 & 2,9 \\
\hline en tallo $(\mathrm{cm})$ & $\mathrm{CP}$ & 3,1 & 3,5 & 3,7 & 3,3 & 3,6 & 3,8 & 3,1 & 4,1 & 3,7 \\
\hline \multirow{2}{*}{\multicolumn{2}{|c|}{$\begin{array}{l}\text { Número de nudos } \\
\text { en rama secundaria } \mathrm{C}\end{array}$}} & 16,7 & 16,7 & 14,1 & 12,7 & 14,3 & 13,1 & 14,4 & 16,1 & 14,0 \\
\hline & & 9,5 & 9,5 & 8,8 & 9,3 & 11,0 & 8,0 & 7,3 & 8,5 & 7,3 \\
\hline \multirow{2}{*}{$\begin{array}{l}\text { Largo de internudos } \\
\text { en rama } \\
\text { secundaria }(\mathrm{cm})\end{array}$} & SP & $1,6 \mathrm{~b}$ & $1,9 \mathrm{~b}$ & $2,3 \mathrm{a}$ & $1,7 \mathrm{~b}$ & $1,8 \mathrm{ab}$ & $2,3 \mathrm{a}$ & 1,7 & 1,8 & 2,1 \\
\hline & $\mathrm{CP}$ & $2,3 \mathrm{~b}$ & $2,1 \mathrm{~b}$ & $2,6 \mathrm{a}$ & $2,2 \mathrm{~b}$ & $2,5 \mathrm{ab}$ & $2,6 \mathrm{a}$ & 2,2 & 1,9 & 2,3 \\
\hline \multirow{2}{*}{$\begin{array}{l}\text { Número de hojas } \\
\text { en rama secundaria }\end{array}$} & SP & 68,0 & 108,9 & 66,9 & 53,4 & 80,6 & 61,4 & 64,9 & 81,4 & 68,3 \\
\hline & $\mathrm{CP}$ & 26,0 & 31,0 & 48,5 & 28,0 & 43,5 & 40,5 & 23,0 & 21,5 & 30,0 \\
\hline \multirow{2}{*}{$\begin{array}{l}\text { Diámetro superior } \\
\text { del tallo }(\mathrm{cm})\end{array}$} & SP & 0,3 & 0,4 & 0,3 & 0,3 & 0,3 & 0,3 & 0,3 & 0,3 & 0,3 \\
\hline & $\mathrm{CP}$ & 0,3 & 0,2 & 0,4 & 0,2 & 0,3 & 0,3 & 0,2 & 0,2 & 0,2 \\
\hline \multirow{2}{*}{$\begin{array}{l}\text { Diámetro } \\
\text { intermedio } \\
\text { del tallo }(\mathrm{cm})\end{array}$} & SP & 0,6 & 0,7 & 0,6 & 0,6 & 0,6 & 0,7 & 0,6 & 0,6 & 0,6 \\
\hline & $\mathrm{CP}$ & 0,5 & 0,6 & 0,6 & 0,5 & 0,6 & 0,6 & 0,4 & 0,5 & 0,5 \\
\hline \multirow{2}{*}{$\begin{array}{l}\text { Diámetro inferior } \\
\text { del tallo }(\mathrm{cm})\end{array}$} & SP & 1,7 & 2,0 & 1,5 & 1,4 & 1,8 & 1,4 & 1,3 & 1,4 & 1,2 \\
\hline & $\mathrm{CP}$ & 1,0 & 1,0 & 1,1 & 0,8 & 1,0 & 0,9 & 0,7 & 0,8 & 0,8 \\
\hline
\end{tabular}

${ }^{1}$ Diferentes letras en la fila indican diferencia significativa $(P \leq 0,05)$ entre procedencias.

2 Different letters on the row indicate significant difference $(P \leq 0,05)$ among provenances.

${ }^{3}$ Procedencia: N: Norte, C: Centro, S: Sur.

4 Tratamiento: SP: sin poda, CP: con poda.

para la procedencia Norte y Centro. Sin embargo, mientras que el efecto de la poda en la procedencia Norte se traduce en un aumento de las dimensiones de la hoja extendida $(1,8 \mathrm{~cm}$ de ancho $\mathrm{x}$ $3,8 \mathrm{~cm}$ de largo las no podadas versus $2,2 \times 4,0$ $\mathrm{cm}$ las podadas), en la procedencia Sur se invierte este efecto (cuadro 3), disminuyendo el tamaño de las hojas en las plantas podadas $(2,3 \times 4,1 \mathrm{~cm}$ versus $2,1 \times 3,7 \mathrm{~cm})$. Podría pensarse que existe una tendencia a compensar la disminución de área foliar debido al menor número de hojas formadas por efecto de la poda (ver variable Número de hojas en rama secundaria, cuadro 1), con un aumento del área foliar de cada hoja en las plantas de la procedencia Norte y en menor grado en las plantas de la procedencia Centro.

El enrollamiento del borde de las hojas es una estrategia que muchas plantas adoptan en respuesta 


\section{CUADRO 2}

Resultados de los análisis de varianza de las variables medidas sobre los tres tallos dominantes de cada individuo.

ANOVA results for variables measured on the three dominant shoots of each individual.

\begin{tabular}{|c|c|c|c|c|c|c|c|c|c|}
\hline \multirow[b]{2}{*}{ Variable } & \multicolumn{3}{|c|}{$1^{\mathrm{er}}$ tallo dominante } & \multicolumn{3}{|c|}{$2^{\circ}$ tallo dominante } & \multicolumn{3}{|c|}{$3^{\text {er }}$ tallo dominante } \\
\hline & $\mathrm{I}^{1}$ & $\mathrm{P}$ & $\mathrm{T}$ & I & $\mathrm{P}$ & $\mathrm{T}$ & I & $\mathrm{P}$ & $\mathrm{T}$ \\
\hline $\begin{array}{l}\text { Largo total } \\
\text { de tallo }\end{array}$ & - & - & $\begin{array}{c}c^{2} \\
0,0007\end{array}$ & - & - & $\begin{array}{c}\mathrm{c} \\
0,0011\end{array}$ & - & - & $\begin{array}{c}\mathrm{c} \\
0,0034\end{array}$ \\
\hline $\begin{array}{l}\text { Número de } \\
\text { ramificaciones } \\
\text { secundarias }\end{array}$ & - & - & - & - & - & - & - & - & $\begin{array}{c}\mathrm{m} \\
0,0213\end{array}$ \\
\hline $\begin{array}{l}\text { Longitud de } \\
\text { rama secundaria }\end{array}$ & - & - & $\underset{0,0104}{\mathrm{~m}}$ & - & - & - & - & - & $\begin{array}{c}\mathrm{c} \\
0,0003\end{array}$ \\
\hline $\begin{array}{l}\text { Número de nudos } \\
\text { en tallo }\end{array}$ & - & - & $\begin{array}{c}\mathrm{c} \\
0,0022\end{array}$ & - & - & $\begin{array}{c}\mathrm{m} \\
0,0204\end{array}$ & - & - & $\begin{array}{c}\mathrm{c} \\
0,0012\end{array}$ \\
\hline $\begin{array}{l}\text { Largo de internudos } \\
\text { en tallo }\end{array}$ & - & - & $\begin{array}{c}\mathrm{c} \\
0,0037\end{array}$ & - & - & $\begin{array}{c}\mathrm{m} \\
0,0445\end{array}$ & - & - & $\begin{array}{c}\mathrm{c} \\
0,0008\end{array}$ \\
\hline $\begin{array}{l}\text { Número de } \\
\text { nudos en rama } \\
\text { secundaria }\end{array}$ & - & - & $\begin{array}{c}\mathrm{c} \\
0,0011\end{array}$ & - & - & $\begin{array}{c}\mathrm{m} \\
0,0154\end{array}$ & - & - & $\begin{array}{c}\mathrm{c} \\
0,0001\end{array}$ \\
\hline $\begin{array}{l}\text { Largo de internudos } \\
\text { en rama secundaria }\end{array}$ & - & $\begin{array}{c}\mathrm{m} \\
0,0117\end{array}$ & $\underset{0,0128}{m}$ & - & $\begin{array}{c}\mathrm{c} \\
0,002\end{array}$ & $\begin{array}{c}\mathrm{m} \\
0,0162\end{array}$ & - & - & - \\
\hline $\begin{array}{l}\text { Número de hojas } \\
\text { en rama secundaria }\end{array}$ & - & - & $\begin{array}{c}\mathrm{c} \\
0,0008\end{array}$ & - & - & - & - & - & $\begin{array}{c}\mathrm{c} \\
0,0012\end{array}$ \\
\hline $\begin{array}{l}\text { Diámetro superior } \\
\text { del tallo }\end{array}$ & - & - & - & - & - & - & - & - & $\begin{array}{c}\mathrm{c} \\
0,0021\end{array}$ \\
\hline $\begin{array}{l}\text { Diámetro intermedio } \\
\text { del tallo }\end{array}$ & - & - & - & - & - & - & - & - & $\underset{0,0454}{\mathrm{~m}}$ \\
\hline $\begin{array}{l}\text { Diámetro inferior } \\
\text { del tallo }\end{array}$ & - & - & $\begin{array}{c}\mathrm{c} \\
0,0001\end{array}$ & - & - & $\begin{array}{c}\mathrm{c} \\
0,0002\end{array}$ & - & - & $\begin{array}{c}c \\
0,0001\end{array}$ \\
\hline
\end{tabular}

${ }^{1}$ Efectos: I: Interacción, P: Procedencia, T: Tratamiento.

${ }^{2}$ Evidencia: c: convincente, m: moderada (24).

a un estrés ambiental, especialmente al déficit hídrico (29). De esta forma se logra reducir la superficie transpirante. En el caso del boldo, las hojas más expuestas (hojas de sol) presentan esta característica a diferencia de las hojas menos expuestas (hojas de sombra, 16). Según los resultados (cuadro 4), esta característica, además de ser afectada por la procedencia, es influenciada por la poda. Las plantas de las procedencias Centro y Sur presentaron un porcentaje de Enrollamiento en ancho de hoja significativamente mayor que las plantas de la procedencia Norte $(57,0 \%$ y
$54,7 \%$ contra $43,2 \%$ para las plantas no podadas y 66,1 y 61,2 contra 54,9 para las plantas podadas; cuadro 3). El tratamiento de poda afectó significativamente este carácter, constatándose un mayor porcentaje de enrollamiento en las plantas intervenidas (cuadro 3).

La variable Curvatura en largo de hoja corresponde a una leve curvatura cóncava, a lo largo de la nervadura central del haz de las hojas. Esta variable evidenció una interacción moderada entre procedencia y poda (cuadro 4), indicando que, dependiendo de la procedencia, el ambiente 


\section{CUADRO 3}

Respuestas medias de las variables medidas en las hojas y de la variable número de rebrotes de tocón para cada procedencia ${ }^{1}$.

Mean responses to the variables measured on the leaves and to the variable number of sprouts of each provenance ${ }^{2}$.

\begin{tabular}{|c|c|c|c|c|}
\hline & & \multicolumn{3}{|c|}{ Procedencia $^{3}$} \\
\hline \multicolumn{2}{|l|}{ Variable } & $\overline{\mathrm{N}}$ & $\mathrm{C}$ & $\mathrm{S}$ \\
\hline \multirow{2}{*}{$\begin{array}{l}\text { Número de rebrotes } \\
\text { de tocón }\end{array}$} & $\mathrm{SP}^{4}$ & $7,3 * *$ & $9,3 * *$ & $9,3 * *$ \\
\hline & $\mathrm{CP}$ & $6,3 *$ & $5,3 *$ & $7,5 *$ \\
\hline \multirow{2}{*}{$\begin{array}{l}\text { Ancho de hoja extendida } \\
(\mathrm{cm})\end{array}$} & $\mathrm{SP}$ & $1,8 \mathrm{~b}$ & $2,2 \mathrm{a}$ & $2,3 \mathrm{a}$ \\
\hline & $\mathrm{CP}$ & 2,2 & 2,2 & 2,1 \\
\hline \multirow{2}{*}{$\begin{array}{l}\text { Largo de hoja extendida } \\
(\mathrm{cm})\end{array}$} & SP & $3,8 \mathrm{~b}$ & $3,6 \mathrm{c}$ & $4,1 \mathrm{a}$ \\
\hline & $\mathrm{CP}$ & $4,0 \mathrm{a}$ & $3,8 \mathrm{ab}$ & $3,7 \mathrm{~b}$ \\
\hline \multirow{2}{*}{$\begin{array}{l}\text { Enrollamiento en ancho } \\
\text { de hoja }(\%)\end{array}$} & SP & $43,2 \mathrm{~b} *$ & 57,0 a $*$ & $54,7 \mathrm{a} *$ \\
\hline & $\mathrm{CP}$ & $54,9 \mathrm{~b}^{* *}$ & $66,1 \mathrm{a}^{* *}$ & $61,2 \mathrm{a}^{* *}$ \\
\hline \multirow{2}{*}{$\begin{array}{l}\text { Curvatura en largo } \\
\text { de hoja }(\%)\end{array}$} & SP & 1,1 & 0,8 & 1,6 \\
\hline & $\mathrm{CP}$ & $2,1 \mathrm{a}$ & $0,8 \mathrm{~b}$ & $1,0 \mathrm{~b}$ \\
\hline \multirow{2}{*}{$\begin{array}{l}\text { Densidad de tricomas en el } \\
\text { haz de la hoja }\left(\mathrm{n}^{\circ} / \mathrm{mm}^{2}\right)\end{array}$} & SP & $4,4 \mathrm{~b} *$ & $5,1 \mathrm{ab} *$ & $5,8 \mathrm{a}$ \\
\hline & $\mathrm{CP}$ & $6,9 * *$ & $6,1 * *$ & 6,2 \\
\hline \multirow{2}{*}{$\begin{array}{l}\text { Densidad de tricomas en el } \\
\text { envés de la hoja }\left(\mathrm{n}^{\circ} / \mathrm{mm}^{2}\right)\end{array}$} & SP & $6,3 b *$ & $10,3 \mathrm{a}$ & 9,8 a $*$ \\
\hline & $\mathrm{CP}$ & $9,8 * *$ & 10,8 & $11,5 * *$ \\
\hline
\end{tabular}

1 Diferentes letras en la fila indican diferencia significativa $(P \leq 0,05)$ entre procedencias. Diferente cantidad de asteriscos en la columna indican diferencia significativa $(P \leq 0,05)$ entre tratamientos.

2 Different letters in the row indicate significant difference $(P \leq 0,05)$ among provenance. Different amount of asterisks in the column indicate significant difference $(P \leq 0,05)$ between treatments.

3 Procedencia: N: Norte, C: Centro, S: Sur.

4 Tratamiento: SP: sin poda, CP: con poda.

modula de diferente forma la respuesta. El porcentaje de Curvatura en largo de hoja de las plantas podadas de la procedencia Norte resultó significativamente mayor que el de las otras procedencias (cuadro 3).

Los tricomas de la superficie foliar también actúan muchas veces como adaptación de los vegetales a ambientes secos (29). Con la presencia de tricomas se logra aumentar la resistencia de la capa límite de la atmósfera en contacto con la superficie de la hoja, reduciendo de esta manera su transpiración. El estudio evidenció una interacción moderada entre la procedencia y el tratamiento en la Densidad de tricomas en el haz $y$ en el envés de la hoja (cuadro 4), indicando que el ambiente modula de diferente forma la respuesta de cada procedencia. Las plantas no podadas de la procedencia Sur presentaron un mayor número de tricomas en el haz de las hojas por unidad de superficie foliar que las plantas no podadas de la procedencia Norte $(5,8$ contra 4,4 tricomas $/ \mathrm{mm}^{2}$ ). Por otra parte, las plantas no podadas de las procedencias Centro y Sur superaron a las plantas no podadas de la procedencia Norte en la Densidad de tricomas en el envés de la hoja (10,3 y 9,8 contra 6,3 tricomas $\left./ \mathrm{mm}^{2}\right)$. El tratamiento de poda en todos los casos aumentó la Densidad de tricomas en ambas caras de las hojas (cuadro 3), siendo este aumento significativo para el haz de las procedencias Norte y Centro y para el envés de las procedencias Norte y Sur. 


\section{CUADRO 4}

Resultados de los análisis de varianza de las variables medidas en las hojas y de la variable número de rebrotes de tocón.

ANOVA results for variables measured on the leaves and for the variable number of sprouts of each individual.

\begin{tabular}{|lccc|}
\hline & \multicolumn{3}{c}{ Efecto } \\
\cline { 2 - 4 } Variable & Interacción & Procedencia & Tratamiento \\
Número de rebrotes & & - & $\mathrm{c}^{1}$ \\
de tocón & - & - & 0,0056 \\
\hline $\begin{array}{l}\text { Ancho de hoja } \\
\text { extendida }(\mathrm{cm})\end{array}$ & $\mathrm{c}$ & - \\
\hline $\begin{array}{l}\text { Largo de hoja } \\
\text { extendida }(\mathrm{cm})\end{array}$ & 0,0001 & - \\
\hline $\begin{array}{l}\text { Enrollamiento en } \\
\text { ancho de hoja }(\%)\end{array}$ & $\mathrm{c}$ & $\mathrm{c}$ \\
\hline $\begin{array}{l}\text { Curvatura en largo } \\
\text { de hoja }(\%)\end{array}$ & 0,0001 & - \\
\hline $\begin{array}{l}\text { Densidad de tricomas } \\
\text { en haz de la hoja }\left(\mathrm{n} \% \mathrm{~mm}^{2}\right)\end{array}$ & 0,0001 & - & - \\
\hline $\begin{array}{l}\text { Densidad de tricomas en } \\
\text { envés de la hoja }\left(\mathrm{n}^{\circ} / \mathrm{mm}^{2}\right)\end{array}$ & $\mathrm{m}$ & - & - \\
\hline
\end{tabular}

${ }^{1}$ Evidencia: c: convincente, m: moderada (24).

A simple vista, también la coloración de las plantas difiere entre procedencias. Así las plantas de la procedencia Sur presentaban una coloración más clara que las plantas de la procedencia Norte. Sin embargo, respecto de los caracteres Color del haz y Color del envés de las hojas (cuadros 5), sólo el color del envés de las hojas analizadas mostró evidencia moderada de asociación del color a la procedencia $(p=0,0145)$, no existiendo asociación significativa entre el color y la procedencia para el haz de las hojas.

Teniendo en cuenta que la procedencia Norte y Sur descienden de poblaciones que se desarrollaron en dos ambientes bioclimáticos diferentes (mesomediterráneo con 400 a 500 mm de precipitaciones y 7 meses de déficit hídrico estival y submediterráneo con 1.200 a $1.300 \mathrm{~mm}$ y menos de 1 mes de déficit hídrico respectivamente) (5), es de esperar que la composición genética actual de ambas poblaciones difiera entre sí. Este hecho aportaría una explicación a las diferencias reportadas entre las procedencias. Así el hábito más compacto y el menor tamaño de hoja de la procedencia Norte daría cuenta de su adaptación a condiciones de mayor déficit hídrico en su ambiente natural, mientras que el mayor enrollamiento de las hojas y la mayor densidad de tricomas en las hojas de la procedencia Sur podrían ser la respuesta al ambiente más severo de la plantación respecto de su hábitat natural. Es de notar que la procedencia Centro ocupa una posición intermedia entre las dos posiciones más extremas. Por otro lado, la respuesta de estas variables al tratamiento de poda confirma que la expresión de estas variables es modulada diferencialmente por el ambiente.

\section{CONCLUSIONES}

Según los resultados obtenidos en este estudio, se puede concluir que las características morfológicas asociadas a la arquitectura de la planta joven en las tres procedencias analizadas de 


\section{CUADRO 5}

Tabla de frecuencia para los colores registrados en el haz y en el envés de las hojas de cada procedencia. Frequency table for the colors registered on the upper and the under leaf side of each origin.

\begin{tabular}{|clccc|}
\hline \multirow{3}{*}{ Color $^{1}$} & & \multicolumn{3}{c|}{ Procedencia } \\
\cline { 3 - 5 } Haz & & Norte & Centro & Sur \\
& & & 11 & 12 \\
& GG 137A & 2 & 4 & 1 \\
& GG 137B & 2 & 8 & 16 \\
& YGG 146A & 11 & 0 & 0 \\
& YGG 146B & 2 & 10 & 4 \\
\hline \multirow{6}{*}{ Envés } & YGG 147A & 11 & 0 & 1 \\
& GG 137D & 0 & 0 & 2 \\
& GG 138A & 0 & 11 & 12 \\
& YGG 144A & 8 & 4 & 7 \\
& YGG 144C & 13 & 1 & 0 \\
& YGG 146B & 0 & 6 & 3 \\
& YGG 146C & 9 & 11 & 8 \\
& YGG 146D & 2 & 0 & 0 \\
\hline
\end{tabular}

${ }^{1}$ RHS Color Chart. The Royal Horticultural Society. London. 1995.

boldo son principalmente moduladas por el medio ambiente. Por otro lado, el tratamiento de poda modifica la arquitectura de la planta, lo que reviste especial importancia si se pretende manejar el boldo como un cultivo intensivo con material seleccionado, favoreciendo una mayor producción de hojas jóvenes en detrimento de los tallos (22).

Las características morfológicas de las hojas de las tres procedencias (color del envés, tamaño de la hoja, enrollamiento del borde y densidad de tricomas en la superficie foliar) obedecen a genotipos distintos, pero cuya expresión es modulada diferencialmente por el ambiente. Por lo tanto sería factible encarar planes de mejoramiento del boldo que junto con buscar una concentración favorable de principios activos (22) logren mejorar aspectos de calidad asociados la presentación del producto cosechado, como color, tamaño y sensación al tacto.

\section{AGRADECIMIENTOS}

Los autores agradecen a Hermine Vogel la posibilidad de acceder a la plantación de procedencias, para la realización de las mediciones.

\section{BIBLIOGRAFIA}

(1) RODRIGUEZ, R., O. MATTHEI, M. QUEZADA. Flora arbórea de Chile. Concepción: Ed. Universidad de Concepción, 1983, 408 p.

(2) RAMIREZ, C., S. LABBE, C. SAN MARTIN, H. FIGUEROA. Sinecología de los bosques de boldo (Peumus boldus) de la cuenca del río Bueno. Bosque (Chile), 1990, vol. 11, No 1, p. 45-56.

(3) DEL FIERRO, P., L. PANCEL. Experiencia silvicultural del bosque nativo de Chile. Santiago: Publicaciones Lo Castillo, 1998, $420 \mathrm{p}$.

(4) GAJARDO, R. La vegetación natural de Chile. Clasificación y distribución geográfica. Santiago: Editorial Universitaria, 1994, $165 \mathrm{p}$.

(5) SAN MARTIN, J. y U. DOLL. Peumus boldus Mol. (Monimiaceae, Magnoliopsida), una especie silvestre promisoria de Chile. Stud. Bot. (España), 1998, vol. 17, p. 109-118.

(6) TORAL, M., U. KANNEGIESSER, R. ROSENDE. Biomasa y boldina en Boldo (Peumus boldus Mol). Ciencia e Investigación Forestal (Chile), 1988, vol. 4, p. 15-25.

(7) ROACH, F. Análisis prospectivo del mercado de hojas de boldo (Peumus boldus Mol.) y sus posibilidades de desarrollo. Tesis. Universidad de Chile, 2001, 87 p.

(8) MUÑOZ, O., M. MONTES, T. WILKOMIRSKY. Plantas medicinales de uso en Chile. Química y Farmacología. Santiago: Ed. Universitaria, 1999, 330 p.

(9) VILA, R., L. VALENZUELA, H. BELLO, S. CANIGUERAL, M. MONTES, T. ADZET. Composition and antimicrobial activity of essential oil of Peumus boldus leaves. Planta Medica (Alemania), 1999, vol. 65, $\mathrm{N}^{\circ} 2$, p. $178-179$.

(10) JIMENEZ, I., A. GARRIDO, R. BANNACH, M. GOTTELAND, H. SPEISKY. Protective effect of boldine against free radical-induced erythrocyte lysis. Phytotherapy Research (USA), 2000, vol, 14, N ${ }^{\circ}$ 5, 339-343.

(11) JANG, Y., J. SONG, Y. SHIN, E. HAN, C. LEE. Protective effect of boldine on oxidative mitochondrial damage in streptozotocin-induced enzymes diabetic rats. Pharmacological Research (USA), 2000, vol. 42, No 4, p. 361-371.

(12) KUBINOVA, R., M. MACHALA, K. MINKSOVA, J. NECA, V. SUCHY. Chemoprotective activity of boldine: modulation of drug-metabolizing enzymes. Pharmazie (Alemania), 2001, Vol. 56, No 3 , p. 242-243.

(13) SCHMEDA-HIRSCHMANN, G., R. RODRIGUEZ, C. THEODULOZ, S. ASTUDILLO, G. FERESIN, A. TAPIA. Free-radical scavengers and antioxidants from Peumus boldus Mol. ("Boldo"). Free radical research (U. K.), 2003, vol. 37, $\mathrm{N}^{\circ}$ 4, p. 447-452.

(14) VOGEL, H., I. RAZMILIC, U. DOLL, R. RUIZ. Variability of some active compounds in boldo (Peumus boldus Mol.). Beiträge zur Züchtungsforschung (Alemania), 1996, vol. 2, $\mathrm{N}^{\circ} 1$, p. 364-367.

(15) VOGEL, H., I. RAZMILIC, U. DOLL. Contenido de aceite esencial y alcaloides en diferentes poblaciones de boldo (Peumus boldus Mol.). Ciencia e investigación agraria (Chile), 1997, vol. 24, $\mathrm{N}^{\text {o }} 1$, p. 1-6. 
(16) VOGEL, H., U. DOLL, M. MUÑOZ, I. RAZMILIC, J. SAN MARTIN, G. VIZCARRA. Boldo (Peumus boldus Mol.) - Vermehrungsversuche und ökophysiologische Studien am natürlichen Standort in Chile. Drogenreport (Alemania), 1998, vol. 11, No 19, p. 14-17.

(17) SCHNEEBERGER, R. Efecto de poda invernal e intensidad de luz sobre el crecimiento y concentración de principios activos en boldo (Peumus boldus Mol.) bajo cultivo. Memoria de título. Universidad de Talca, 2001.

(18) BERRÍOS, C. Efecto de la densidad de plantación y dos niveles de riego sobre el rendimiento y los principios activos en boldo (Peumus boldus Mol). Memoria de título. Universidad de Talca, 2003.

(19) GUERRA, M. Variación genética en el contenido de alcaloides y aceite esencial en boldo (Peumus boldus Mol). Tesis. Universidad de Talca, 1998.

(20) VOGEL, H., I. RAZMILIC, M. MUÑOZ, U. DOLL, J. SAN MARTIN. Studies of genetic variation of essential oil and alkaloid content in boldo (Peumus boldus Mol.). Planta Medica (Alemania), 1999, vol. 65, p. 90-91.

(21) ACEVEDO, P. Variabilidad en los principios activos de tres poblaciones de boldo (Peumus boldus Mol). Tesis de Magíster. Universidad de Talca. 2003.

(22) VOGEL, H., I. RAZMILIC, J. SAN MARTIN, U. DOLL y B. GONZALES. Plantas medicinales chilenas. Experiencias de Domesticación y Cultivo de Boldo, Matico,
Bailahuén, Canelo, Peumo y Maqui. Talca: Ed. Universidad de Talca, 2005. Capítulo 3. Boldo, p. 23-54.

(23) DIRECCION METEOROLOGICA DE CHILE. Séptima Región. Descripción Climatológica. [citado 14 julio 2005]. Disponible en: http://www.meteochile.cl/ climas/climas_septima region.html.

(24) RAMSEY, F., D. SCHAFER. The Statistical Sleuth. A Course in Methods of Data Analysis. Belmont: Wadsworth Publishing Company, 1997. Interpretation of p-Values, p. 44-45.

(25) MONTGOMERY, D. Diseño y Análisis de Experimentos. México: Ed. Iberoamericana, 1991, 569 p.

(26) OEHLERT, G. First Course in Design and Analysis of Experiments. New York: W. H. Freeman and Company. 2000. Transformation of the response, p. 126-127.

(27) HARTUNG, J., B. ELPELT y K.-H. KLÖSENER. Statistik. Lehr- und Handbuch der angewandten Statistik. Munich: Ed. R., Oldenbourg, 1986. Assoziationsmasse in allgemeinen 2-dimensionalen Kontingenztafeln, p. 450-464.

(28) BARTHELEMY, D. Architecture et sexualité chez quelques plantes tropicales: le concept de floraison automatique. Tesis. Université de Sciences et Techniques de Languedoc. Académie de Montpellier, 1988, 262 p.

(29) LARCHER, W. Ökophysiologie der Pflanzen. Leben, Leistung und Stressbewältigung der Pflanzen in ihrer Umwelt. Regensburg: Ed. Eugen Ulmer GmbH \& Co., 1994. Überleben bei Dürre, p. 306-312. 